\title{
EyeSys corneal topography measurement applied to calibrated ellipsoidal convex surfaces
}

\author{
William Arthur Douthwaite
}

\begin{abstract}
Aims/Background-This study was carried out to assess the accuracy of the EyeSys videokeratoscope by using convex ellipsoidal surfaces of known form.

Methods-PMMA convex ellipsoidal buttons were calibrated using Form Talysurf analysis which allowed subsequent calculation of the vertex radius and $p$ value of the surface. The EyeSys videokeratoscope was used to examine the same ellipsoids. The tabular data provided by the instrument software were used to plot a graph of $r^{2}$ versus $y^{2}$ where $r$ is the measured radius at $y$, the distance from the corneal point being measured to the surface vertex. The intercept on the ordinate of this graph gives the vertex radius and the slope the $p$ value. The results arising from the Talysurf and the EyeSys techniques were compared.
\end{abstract}

Results-The EyeSys videokeratoscope gave readings for both vertex radius and $p$ value that were higher than those of the Talysurf analysis. The vertex radius was around $0.1 \mathrm{~mm}$ greater. The $p$ value results were similar by the two methods for $p$ values around unity but the EyeSys results were higher and the discrepancy increased as the $p$ value approached that of a paraboloid.

Conclusions-Although the videokeratoscope may be useful in comparative studies of the cornea, there must be some doubt about the absolute values displayed. The disagreement is sufficiently large to suggest that the instrument may not be accurate enough for contact lens fitting purposes.

(Br f Ophthalmol 1995; 79: 797-801)

Corneal topographic examination has become a popular technique for monitoring corneal changes after various types of ocular surgery. It is also being used as a way of assessing the after effects of contact lens wear. These studies are comparative in nature and in consequence the accuracy of the values of corneal radius are not crucial as long as the precision (repeatability) of the results is good. However, the instrument manufacturers are now offering contact lens fitting software which takes the corneal curvature values, measured by the instrument, and uses these to calculate a contact lens back surface geometry to provide an optimum fit. The absolute values measured by the instrument now become critical and so the accuracy of topographic corneal analysis systems should be assessed on surfaces similar to those of the typical cornea.

The keratoscope target consists of a number of concentric rings. These must be positioned so that the image of every ring is simultaneously in focus in the plane of the photokeratoscope camera film or the equivalent for the video camera of a videokeratoscope. The design of the target must be based on the assumption that it is going to measure a specific surface. The ring images will all be simultaneously clear when this surface is being measured. However, when a steeper or flatter surface, or a surface which is more aspherical, is measured then some of the ring images will not be focused clearly and the accuracy of the resulting measurement will suffer.

Furthermore, the computerised instruments use automated calculation of the corneal topography and, for proprietary reasons, the manufacturers do not indicate the method used. It is thus impossible to know what assumptions and/or approximations have been made in writing the computer programs which are used to analyse the data. It is admitted, for example, that the early algorithms made assumptions which can only be applied to spherical surfaces, when there is general agreement that the normal cornea approximates to a prolate (flattening) ellipsoid. ${ }^{12}$ Wang et $a l^{3}$ proposed an improved algorithm that avoids some of the earlier assumptions and this produced identical results for spherical surfaces but substantially improved accuracy for aspherical surfaces at the cost of increased computation time. It must be noted, however, that the experimental procedure involved the use of only one steel sphere (radius $8.33 \mathrm{~mm}$ ) and one plastic ellipsoid (vertex radius $7 \cdot 73$ (SD 0.02) $\mathrm{mm}$ and eccentricity 0.5 ).

A review paper by Morrow and Stein ${ }^{4}$ summarises the development of keratoscopy which ends with the description of the two currently most popular clinical video keratoscopes. These are the EyeSys corneal analysis system (EyeSys Laboratories, Houston, TX, USA) and the TMS-1 topographic modelling system (Computed Anatomy Inc, NY, USA). 
These two instruments were compared by Antalis $e t a l^{5}$ using patients with keratoconus, corneal scars, and residual astigmatism following refractive surgery. They noted that the two systems performed equally well and were similar in application and shortcomings. They also noted that some of the shortcomings are being solved by software improvement. Maquire et al ${ }^{6}$ devised a computer program to make reproducibility calculations for corneal topography systems. The EyeSys and the TMS-1 were compared for their reproducibility on three spheres. It was noted that the EyeSys displayed more variability in readings close to the keratoscopic pole than the TMS-1. Both systems performed equally well for all semi-meridians.

In general, the assessment of instrument performance has been conducted by using either calibrated spherical surfaces or human corneas where surface calibration is, of course, not possible. The human cornea approximates to a prolate (flattening) ellipsoid. ${ }^{12}$ The vertex (apical) radius will be around $7.8 \mathrm{~mm}$ and the $\mathrm{p}$ value around 0.8 in an average cornea. The $p$ value $(p)$ is a variable which indicates the rate of change of radius of curvature from the vertex region to the periphery. Its use is more appropriate, in dealing with the cornea, than the eccentricity $(e)$ because the $p$ value will describe the oblate (steepening) ellipsoid, which the eccentricity will not, and a not insignificant number of normal human corneas take the oblate ellipsoidal form. ${ }^{7} \mathrm{~A} p$ value of zero indicates a paraboloidal surface. A p value of unity indicates a spherical surface. The prolate ellipsoids lie between these limits. A $p$ value greater than unity indicates the presence of an oblate ellipsoid. A negative $p$ value denotes a hyperboloidal surface.

This work describes the assessment of the performance of the EyeSys instrument on convex ellipsoidal surfaces. These ellipsoids were calibrated by using the Form Talysurf analysis technique which allows subsequent calculation of the vertex radius and the $p$ value. These results were compared with the vertex radius and $p$ value acquired by the EyeSys instrument.

\section{Materials and methods}

Twenty four conicoidal convex plastic buttons were manufactured and then sent to the Rank Taylor Hobson Calibration and Measurement Laboratory for Form Talysurf analysis. This laboratory is part of the National Measurement Accreditation Service which provides facilities for checking or calibrating instruments and other test equipment against recognised international standards. It has been approved for the measurement of roundness, surface finish, and angle in engineering components.

The measurement of the conicoidal buttons was made at $20^{\circ} \mathrm{C}$ using the Form Talysurf measuring instrument, calibrated with reference to traceable standards. The instrument utilises a computer controlled stylus which traverses any given meridian of the surface. A laser interferometric transducer is used which transmits the signals to a microcomputer for detailed processing. The resolution is quoted as $20 \mathrm{~nm}$ or approximately ${ }^{1 / 32}$ of the wavelength of helium neon laser light. Relative to the best fit arc, the accuracy is claimed to be within two parallel planes having a separation of $0.1 \mu \mathrm{m}$ over a $20 \mathrm{~mm}$ traverse after the removal of the best fit reference line.

Two measurements were made on each lens: one parallel to an engraved line on the underside of the button and the other at $90^{\circ}$ to the first one. Both measurements were made over the central $10 \mathrm{~mm}$ of the button. Each surface was scanned and the resulting printout indicated the departure from the surface form. The form of the surface was analysed and the major and minor axis semidiameters ( $a$ and $b$ ) were stated for the elliptical cross section. It is then a simple matter to calculate the vertex radius and $p$ value of the ellipse.

$$
\begin{aligned}
& \text { The vertex radius } r_{\mathrm{o}}=\frac{b^{2}}{a} \\
& \text { The } \mathrm{p} \text { value } \quad p=\frac{b^{2}}{a^{2}}
\end{aligned}
$$

where $a$ is the semidiameter of the major axis and $b$ is the semidiameter of the minor axis. The eccentricity $(e)$ of the ellipse can be deduced using:

$$
e=\sqrt{ }(1-p)
$$

The use of the $p$ value allows consideration of steepening curvature (when $p>1$ ), which eccentricity cannot. It is, therefore, intended that $p$ will be used throughout this paper.

The results of this analysis are assumed to give an accurate assessment of the conicoidal surfaces and this provides a datum against which the results of any other method can be compared. The vertex radii ranged from $7 \cdot 131$ to $8.203 \mathrm{~mm}$ and the $\mathrm{p}$ values varied between $0 \cdot 135$ and 0.993 .

The EyeSys corneal analysis instrument (EyeSys Windows Workstation Version 2.00W) was first calibrated by following the routine described in the operator's manual. A calibration check was made on one of the calibration spheres before the investigation commenced and the results were printed out to allow a subsequent analysis. The operator's manual states that the instrument is considered to be in calibration if the measured power of the spherical surface is within $0.25 \mathrm{D}$ of the stated value.

Each aspheric button was placed on a special holder that was made to fit onto the chin rest of the instrument. The engraved line was oriented to a horizontal position. The matt black holder was designed to ensure that the long axis of the ellipsoid was parallel to the optical axis of the instrument. The mounted aspheric buttons reflected a similar amount of light to that of the instrument's own calibration spheres. The manufacturer's recommendations for surface topography measurement were followed, being particularly careful to achieve an accurate centration of the image on the computer monitor screen and to focus the image as precisely as possible because McCarey et al ${ }^{8}$ state that accurate results in 


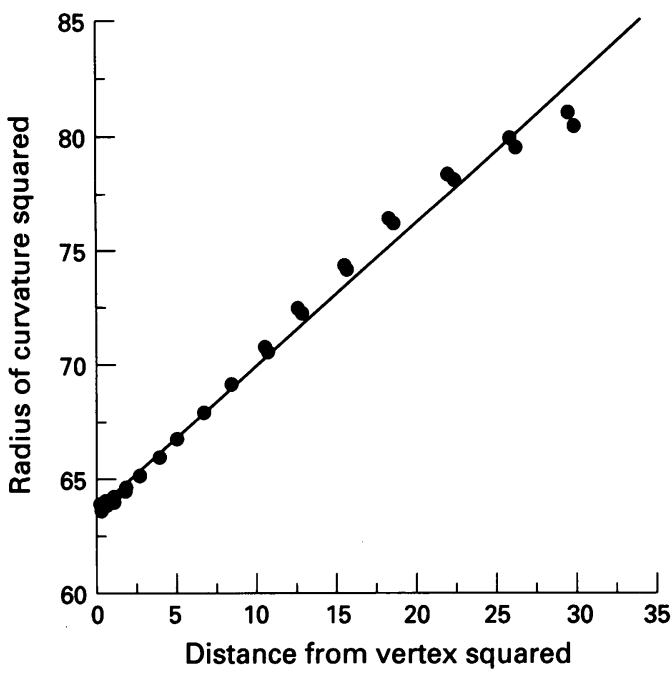

Figure 1 Details of one of the ellipsoidal surfaces. An ellipsoid theoretically produces a linear function when the square of the vertex distance is plotted against the square of the radius of curvature. The regression line predicts a value of $7.983 \mathrm{~mm}$ for the vertex radius and 0.375 for the $p$ value. The regression line is of the form $\mathrm{y}=63 \cdot 734+0.625 \mathrm{x}$.

their investigation were obtainable only with critical focusing and image centralisation. Any possible tilt of the button was monitored by inspecting the tabular display, after image capture, for any asymmetry between the semimeridians. Any asymmetry was minimised by small adjustments of the lens holder, and a new image was captured. The remaining image acceptance criteria adopted were those used by McCarey et al. ${ }^{8}$ These were: (1) the mire image must be centred on the monitor; (2) the focusing aid (a cross centred inside a circular window) must be used to achieve the best focus before the image is captured; (3) the innermost ring must be complete. Editing of the image was not allowed. The image was captured and analysed by the instrument. The tabular display was requested for the horizontal and vertical meridians and this information was printed out in order to allow subsequent analysis of the data. The display gives the radius and location of a series of measurement points in terms of the displacement from the keratoscopic pole along the specified meridian. This information is provided for both sides of the vertex. The results for the meridian at $90^{\circ}$ to the first (both superior and inferior displacements from the vertex) are also specified by the videokeratoscope but these data were not used in this investigation. Ten independent measurements were taken on each button.

Bennett ${ }^{9}$ derived the equation for an elliptical section:

$$
r_{\mathrm{s}}=\sqrt{ }\left[r_{\mathrm{o}}{ }^{2}+(1-p) y^{2}\right]
$$

where $r_{\mathrm{s}}$ is the sagittal radius for a point perpendicular distance $y$ from the major axis of the ellipse, which has a vertex radius $r_{o}$ and a $\mathrm{p}$ value $p$.

Equation (4) can be written as:

$$
r_{\mathrm{s}}^{2}=r_{\mathrm{o}}{ }^{2}+(1-p) y^{2}
$$

and since the vertex radius $r_{\mathrm{o}}$ and the $\mathrm{p}$ value $p$ are constants of the surface, the equation is the familiar equation for a straight line graph. If,

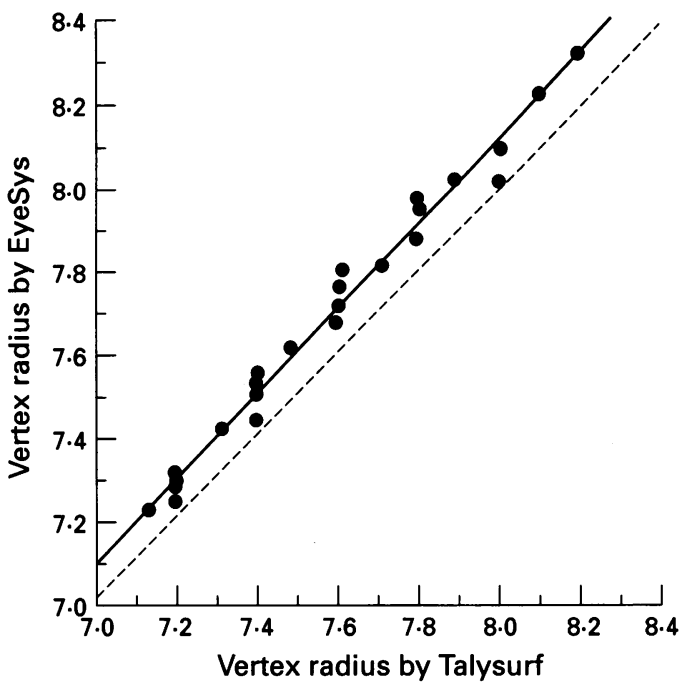

Figure 2 Comparison of the vertex radius results acquired by the Talysurf and the EyeSys techniques. The broken line is the line of equality where $\mathrm{y}=\mathrm{x}$. The regression line is of the form $\mathrm{y}=0.036+1 \cdot 01 \mathrm{x}$.

therefore, we assume that the keratoscope is measuring the sagittal radius of the ellipsoids, as suggested by Bennett and Rabbetts, ${ }^{10}$ then we can plot the tabular data out in a graph of $r_{\mathrm{s}}^{2}$ (on the $\mathrm{y}$ axis versus $y^{2}$ (on the $\mathrm{x}$ axis) and calculate the best fitting line by the method of least squares. The equation for that line will be equation (5), so that the intercept on the $y$ axis gives $r_{\mathrm{o}}{ }^{2}$ and the slope of the line is $1-p$. Figure 1 shows a typical plot of one of the measurements of an ellipsoidal button. Both the temporal and nasal quadrant results were included in the scatterplot. The variables $r_{\mathrm{o}}$ and $p$ were then recorded as a description of the ellipsoidal surface. The 10 results were averaged to give a mean value for the vertex radius and for the $p$ value of each button. These mean values were then compared with the vertex radius and $p$ value determined by the Talysurf analysis.

\section{Results}

The calibration surface (claimed to be a $7.94 \mathrm{~mm}$ radius spherical surface) was analysed using the $r_{\mathrm{s}}^{2}$ versus $y^{2}$ graph and this produced a $\mathrm{y}$ axis intercept that predicts a vertex radius of $7.938 \mathrm{~mm}$ and a slope $(p-1)$ of 0.001 , which means that the $p$ value is 0.999 . Thus the analysis suggests that the surface is almost spherical $(p=1)$ with a radius of curvature of $7.938 \mathrm{~mm}$. This represents a discrepancy of $0.01 \mathrm{D}$ which is well within the acceptable range according to the EyeSys operator's manual and in consequence the instrument was considered to be adequately calibrated.

The aspheric buttons were analysed in the same way, to provide the vertex radius and the $p$ value for each surface. These variables could then be compared with those acquired by the Talysurf analysis procedure.

\section{VERTEX RADIUS}

Figure 2 compares the vertex radius deduced by the two techniques. The regression line is 


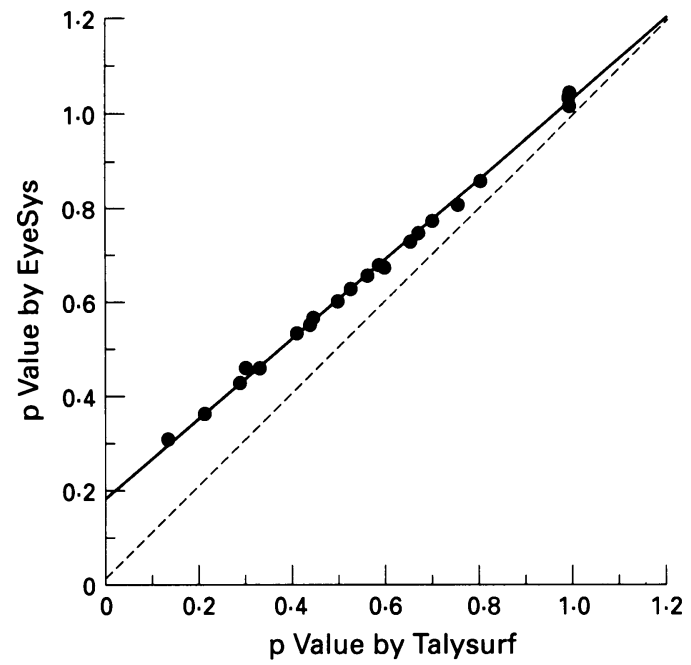

Figure 3 Comparison of the $p$ value acquired by the Talysurf and the EyeSys techniques. The broken line is the line of equality where $\mathrm{y}=\mathrm{x}$. The regression line is of the form $\mathrm{y}=0 \cdot 185+0 \cdot 839 \mathrm{x}$.

approximately parallel to the line of equality $(y=x)$ but it suggests that the EyeSys is overestimating the vertex radius by $0.108 \mathrm{~mm}$ for a Talysurf vertex radius of $7.2 \mathrm{~mm}$ and by $0.118 \mathrm{~mm}$ for a vertex radius of $8.2 \mathrm{~mm}$.

Bland ${ }^{11}$ recommends using the one sample $t$ method for comparing two methods of measurement, providing the data are normally distributed. The distribution of the data was found to be not significantly different from normal using the Kolmogorov Smirnov $d$ test and the $\chi^{2}$ test. If Bland's recommendation is adopted then the EyeSys instrument is seen to overestimate by as much as $0.13 \mathrm{~mm}$ or as little as $0.09 \mathrm{~mm}$ for the range of radii studied, assuming a $95 \%$ confidence interval.

\section{p VALUE}

Figure 3 illustrates the relation between the $p$ values measured by the Talysurf and the EyeSys techniques. Once again the regression line does not coincide with the line of equality. The EyeSys appears to be overestimating a $p$ value of $0 \cdot 2$ by $0 \cdot 153$. However, when the surface is spherical with a $p$ value of unity, the EyeSys overestimates the $\mathrm{p}$ value by only $0 \cdot 024$. Once again the data appear to be normally distributed and the one sample $t$ method for comparing two methods of measurement indicates that the EyeSys could overestimate by as much as $0 \cdot 105$ or as little as 0.067 for the range of $\mathrm{p}$ values studied, assuming a $95 \%$ confidence interval.

\section{REPEATABILITY}

Two aspheric buttons were selected in order to assess the repeatability of the EyeSys measurement. The first button was selected from those already measured and the Talysurf assessment of this surface indicated that the vertex radius was $7.799 \mathrm{~mm}$ with a $p$ value of 0.655 . This specification is close to that of the typical human cornea.

The second button was selected from a group which was not used in the study described above. The EyeSys image focusing end point is achieved by centring a cross inside a small circular window. These two features are observed on the monitor screen at the same time as the ring images themselves. This feature makes focusing very easy and precise. However, when dealing with surfaces which possess flat peripheral curves, the window and the cross are displaced outside the boundaries of the screen. In these circumstances, the operator is advised to attempt to achieve the best focus by observing a pair of small circles between the seventh and eighth rings of the target. the focusing appears to be far less precise in these circumstances and it was therefore suspected that the repeatability could deteriorate when measuring such a surface. With this in mind a button with an aspheric surface, assessed by Talysurf as having a vertex radius of $8.199 \mathrm{~mm}$ and a $\mathrm{p}$ value of 0.183 , was selected for investigation. The $p$ value of this surface indicates that the peripheral radius will be much flatter than that of the vertex. In fact the EyeSys measured the peripheral radius as $9.36 \mathrm{~mm}$ at a diameter of $10 \mathrm{~mm}$ and this resulted in the disappearance of the window and cross required for precise focusing.

Each button was subjected to 10 independent measurements with the button and the button holder being removed from the instrument between measurements.

The data were apparently normally distributed according to the Kolmogorov Smirnov $d$ test and the $\chi^{2}$ test.

The EyeSys mean vertex radius for the first button was $7.876 \mathrm{~mm}$ with a standard deviation of $0.011 \mathrm{~mm}$. The mean $\mathrm{p}$ value was 0.724 with a standard deviation of $0 \cdot 004$.

The EyeSys mean vertex radius for the second button was $7.853 \mathrm{~mm}$ with a standard deviation of $0.062 \mathrm{~mm}$. The mean $p$ value was -0.216 with a standard deviation of 0.243 .

It was obviously possible to acquire a standard deviation for the 10 readings of all the buttons in the main study. The average standard deviation for the 24 buttons was 0.014 for the vertex radius and 0.008 for the $\mathrm{p}$ value.

\section{Discussion}

Assessment of the accuracy of the EyeSys in past papers has been limited, in the main, to the assessment of calibrated spherical surfaces. The normal human cornea approximates to a prolate ellipsoid ${ }^{12}$ and so it is more appropriate to use calibrated ellipsoidal surfaces. In the case of the spherical surface the instrument simply needs to make an accurate assessment of the radius of curvature, which is constant across the surface. Any error in the calculation of the displacement of the image point or region to which the curvature is ascribed will not give rise to an error in the description of the surface - that is, the estimate of the vertex radius and $p$ value will not be affected. This is not the case for the ellipsoid. An error in estimating the position of the point with the stated radius will change the estimated value of both the vertex radius and the $p$ value. 
Van Saarloos and Constable ${ }^{12}$ stated that exact calculations are not possible from the photokeratoscope data and many assumptions are made to obtain an estimate of the corneal topography. They described their system of algorithms which were claimed to produce a result that was seven times more accurate although the computation time was increased by three times.

Wang et $a l^{3}$ used a single aspheric surface which was calibrated with a microscope that used stage verniers to permit measurement in the $x$ and $y$ directions, with the $z$ direction measured by using an objective with a shallow depth of field, focused onto the surface micro structure. This approach suffers from the fact that the calculated $p$ value and vertex radius are sensitive to focusing errors. Also the measurement of a single aspheric surface will not provide a complete picture.

The EyeSys was compared with the TMS-1 by Wilson et al ${ }^{13}$ using calibrated spherical surfaces and normal human corneas. The TMS-1 was found to be statistically more accurate in determining the power of calibrated spheres near the keratoscopic pole and the EyeSys was more accurate at a distance of $3 \mathrm{~mm}$. They concluded that the small difference in accuracy between the two instruments is unlikely to be of clinical significance. The two instruments gave similar results for the corneas of 11 normal subjects.

The problem with ellipsoidal surfaces is that they are unlikely to be manufactured to the exact form requested and must therefore be measured in some precise way. The Form Talysurf technique appears to be the most appropriate way to establish the ellipsoidal surface specification.

The results of this study indicate that the EyeSys instrument overestimates both the vertex radius and the $p$ value when compared with the results of Talysurf analysis. The estimate of the $p$ value may be acceptably accurate in the 0.8 to 1.0 region. However, the error for $p$ values outside this zone and the error in the vertex radius casts doubt on the accuracy of the instrument.

The assumption has been made that the radius values calculated by the instrument are sagittal radius values because Bennett and Rabbetts ${ }^{10}$ deduced that the keratometer measures sagittal radius and the designers of the EyeSys have assumed a sagittal radius measurement. If the radius readings displayed by the EyeSys instrument were considered to be tangential then the calculation of the vertex radius would not change by very much, however, the calculated $\mathrm{p}$ value would be even higher than the values calculated above.

The repeatability exercise leads to the conclusion that the measurement may be acceptably repeatable when the 'cross in the window' focusing aid can be used. If we take two standard deviations as a representation of the spread of the readings then the vertex radius could vary by plus or minus $0.022 \mathrm{~mm}$ and the $\mathrm{p}$ value by plus or minus 0.008 according to the measurements on the first button. However, if the cross in the window aid is not displayed on the monitor screen, the repeatability deteriorates. The two standard deviation values became plus or minus $0.124 \mathrm{~mm}$ for vertex radius and plus or minus 0.486 for the $p$ value when the second aspheric button was examined. It must be also noted that not only was there an increase in the standard deviation here but there was also a substantial difference in the values of the vertex radius (EyeSys under reads by $0.346 \mathrm{~mm}$ ) and the $\mathrm{p}$ value (EyeSys under reads by 0.399 ). It must be emphasised that these figures come from the examination of only two buttons and that variations in both vertex radius and $p$ value have not been considered. However, the results suggest that the accuracy and the precision of an EyeSys measurement are likely to be poor when the focusing aids are not visible on the monitor screen.

The use of calibrated ellipsoidal buttons leads to the conclusion that the current EyeSys corneal analysis system does not produce results which are accurate enough, for example, for the proposal that the calculated form of the cornea can be used to provide the back surface specification for an optimally fitting contact lens. The repeatability of the corneal analysis system may be acceptable and comparative investigations may be successful but there must be a question hanging over the absolute values that are displayed.

1 Mandell RB, StHelen R. Mathematical models of the general corneal contour. $B r \mathcal{F}$ Physiol Opt 1971; 26: 183-97.

2 Burek H, Douthwaite WA. Mathematical models of the general corneal surface. Ophthal Physiol Opt 1993; 13: 86-92.

3 Wang MSJ, Rice DA, Klyce SD. A new reconstruction algorithm for improvement of corneal topographical algorithm for improvement of corneal top.

4 Morrow GL, Stein RM. Evaluation of corneal topography: past, present and future trends. Can $\mathcal{F}$ Ophthalmol 1992 27: 214-25.

5 Antalis J, Lembach RG, Carney LG. A comparison of the TMS-1 and the corneal analysis system for the analysis of abnormal cornea. $C L A O F$ 1993; 19: 58-63.

6 Maquire LJ, Wilson SE, Comp JJ, Verity S. Evaluating the reproducibility of topography systems on spherical surfaces. Arch Ophthalmol 1993; 111: 259-62.

7 Guillon M, Lydon DPM, Wilson C. Corneal topography: a clinical model. Ophthal Physiol Opt 1986; 6: 47-56.

8 McCarey BE, Zurawski CA, O'Shea DS. Practical aspects of a corneal topography system. CLAO $\mathcal{F} 1992$; 18: 248-54.

9 Bennett AG. Aspherical and continuous curve contact lenses. Part Three. Optometry Today 1988; 28: 238-42.

10 Bennett AG, Rabbetts RB. What radius does the conventional keratometer measure? Ophthal Physiol Opt 1991; 11: 239-47.

11 Bland M. An introduction to medical statistics. Oxford: Oxford Medical Publications, 1987: 280-3.

12 Van Saarloos PP, Constable IJ. Improved method for calculation of corneal topography for any keratoscope geometry. Optom Vis Sci 1991; 68: 960-5.

13 Wilson SE, Verity SM, Conger DL. Accuracy and precision of the corneal analysis system and the topographic modeling system. Cornea 1992; 11: 28-35. 